\title{
Development of a targeted sequencing approach to identify prognostic, predictive and diagnostic markers in paediatric solid tumours
}

\author{
Elisa Izquierdo ${ }^{1,2}$, Lina Yuan ${ }^{1}$, Sally George ${ }^{3,4}$, Michael Hubank $^{1}$, Chris Jones ${ }^{2}$, \\ Paula Proszek ${ }^{1}$, Janet Shipley ${ }^{5}$, Susanne A. Gatz ${ }^{4,5}$, Caedyn Stinson ${ }^{6}$, Andrew S. \\ Moore $^{6,7,8}$, Steven C. Clifford9 ${ }^{9}$, Debbie Hicks9 ${ }^{9}$ Janet C. Lindsey ${ }^{9}$, Rebecca M. Hill9, \\ Thomas S. Jacques ${ }^{10,11}$, Jane Chalker ${ }^{12}$, Khin Thway ${ }^{13}$, Simon O'Connor ${ }^{14}$, Lynley \\ Marshall ${ }^{4}$, Lucas Moreno ${ }^{4,15}$, Andrew Pearson ${ }^{4}$, Louis Chesler ${ }^{3,4}$, Brian A. Walker ${ }^{1,16, *}$ \\ and David Gonzalez De Castro ${ }^{1,17, *}$ \\ ${ }^{1}$ Molecular Diagnostics Department, The Institute of Cancer Research and Clinical Genomics, The Royal Marsden NHS \\ Foundation, London, United Kingdom \\ ${ }^{2}$ Glioma Team, Division of Molecular Pathology and Cancer Therapeutics, The Institute of Cancer Research, London, United \\ Kingdom \\ ${ }^{3}$ Paediatric Tumour Biology, Division of Molecular Pathology, The Institute of Cancer Research, London, United Kingdom \\ ${ }^{4}$ Paediatric Drug Development Team, Children and Young People's Unit, Royal Marsden NHS Foundation Trust, London, United \\ Kingdom \\ ${ }^{5}$ Sarcoma Molecular Pathology Team, Divisions of Molecular Pathology and Cancer Therapeutics, The Institute of Cancer \\ Research, London, United Kingdom \\ ${ }^{6}$ The University of Queensland Diamantina Institute, Translational Research Institute, Brisbane, Australia \\ ${ }^{7}$ Oncology Service, Children's Health Queensland Hospital and Health Service, Brisbane, Australia \\ ${ }^{8}$ UQ Child Health Research Centre, The University of Queensland, Brisbane, Australia \\ ${ }^{9}$ Wolfson Childhood Cancer Research Centre, Northern Institute for Cancer Research, Newcastle University, Newcastle Upon \\ Tyne, United Kingdom \\ ${ }^{10}$ Department of Histology, Great Ormond Street Hospital for Children NHS Foundation Trust, London, United Kingdom \\ ${ }^{11}$ Developmental Biology and Cancer Programme, UCL GOS Institute of Child Health, London, United Kingdom \\ ${ }^{12}$ Haematology, Cellular and Molecular Diagnostics Service, UCL GOS Institute of Child Health, London, United Kingdom \\ ${ }^{13}$ Sarcoma Unit, Royal Marsden NHS Foundation Trust, London, United Kingdom \\ ${ }^{14}$ Haemato-Oncology Unit, Royal Marsden NHS Foundation Trust, London, United Kingdom \\ ${ }^{15}$ HNJ-CNIO Clinical Research Unit and Hospital Universitario Niño Jesus, Madrid, Spain \\ ${ }^{16}$ Myeloma Institute, University of Arkansas for Medical Sciences, Little Rock, Arkansas, USA \\ ${ }^{17}$ Centre for Cancer Research and Cell Biology, Queen's University Belfast, Belfast, United Kingdom \\ *These authors have contributed equally to this work \\ Correspondence to: David Gonzalez De Castro, email: D.GonzalezdeCastro@qub.ac.uk \\ Keywords: childhood cancer; targeted sequencing; molecular diagnostics; panel validation; targeted therapies \\ Received: July 28, $2017 \quad$ Accepted: November 16, $2017 \quad$ Published: December 06, 2017
}

Copyright: Izquierdo et al. This is an open-access article distributed under the terms of the Creative Commons Attribution License 3.0 (CC BY 3.0), which permits unrestricted use, distribution, and reproduction in any medium, provided the original author and source are credited.

\section{ABSTRACT}

The implementation of personalised medicine in childhood cancers has been limited by a lack of clinically validated multi-target sequencing approaches specific for paediatric solid tumours. In order to support innovative clinical trials in high-risk patients with unmet need, we have developed a clinically relevant targeted sequencing panel spanning $311 \mathrm{~kb}$ and comprising 78 genes involved in childhood cancers. A total of 132 samples were used for the validation of the panel, including Horizon Discovery 
cell blends $(n=4)$, cell lines $(n=15)$, formalin-fixed paraffin embedded (FFPE, $n=83$ ) and fresh frozen tissue $(F F, n=30)$ patient samples. Cell blends containing known single nucleotide variants $(\mathrm{SNVs}, \mathrm{n}=528)$ and small insertion-deletions (indels $n=108$ ) were used to define panel sensitivities of $\geq 98 \%$ for SNVs and $\geq 83 \%$ for indels [95\% CI] and panel specificity of $\geq 98 \%$ [95\% CI] for SNVs. FFPE samples performed comparably to FF samples ( $n=15$ paired). Of 95 well-characterised genetic abnormalities in 33 clinical specimens and 13 cell lines (including SNVs, indels, amplifications, rearrangements and chromosome losses), 94 (98.9\%) were detected by our approach. We have validated a robust and practical methodology to guide clinical management of children with solid tumours based on their molecular profiles. Our work demonstrates the value of targeted gene sequencing in the development of precision medicine strategies in paediatric oncology.

\section{INTRODUCTION}

Cancer remains the leading cause of death due to disease in children aged $>1$ year [1]. Cure rates for paediatric solid tumours have not substantially improved in the past decade with patients having recurrent disease performing particularly badly, reflecting the limitations of current approaches that employ intensive chemotherapy, surgery and radiation [2-4]. In adults, the stratification of patients by genetic profiling using high throughput sequencing has supported adaptive clinical trials $[5,6]$, and there is an urgent need to translate such opportunities to the treatment of childhood disease.

The genomic landscape of paediatric cancer is becoming increasingly well-defined leading to the conclusion that childhood cancers have in general fewer somatic mutations than adults, but that mutations in epigenetic regulators occur at a higher incidence [717]. Key recent findings include recurrent mutations in the genes encoding histones 3.3 and 3.1 ( $H 3 F 3 A$ and $H I S T 1 H 3 B$ ) as well as the activin A receptor type I $(A C V R 1)$ that are unique to paediatric high-grade glioma (pHGG) and diffuse intrinsic pontine glioma (DIPG) [1820]. Similarly, ATRX mutations, TERT rearrangements and $M Y C N$ amplification define mutually exclusive molecular subgroups of neuroblastoma, all of which are associated with poor prognosis [21-23]. The newly proposed molecular-based medulloblastoma sub-classification defines subgroups, each of which potentially requires a tailored therapeutic strategy [7, 11, 24].

Despite our improved knowledge of somatic alterations in paediatric cancers, precision medicine remains unavailable for the majority of patients. For example, a small number of early-phase paediatric trials are recruiting children whose tumours harbour genetic alterations including $A L K$ genomic alterations (mutations, amplifications or translocations) that can be treated with ALK inhibitors and BRAF V600 mutant tumours that can be treated with BRAF or MEK inhibitors.

In addition, there is now an extensive list of recurrent genetic alterations with potential diagnostic, prognostic or predictive value, and sequential testing of single genes using standard methods has become unfeasible due to lack of available material and high costs. High-throughput sequencing (also known as next generation sequencing or NGS) offers a solution to these issues. In particular, panel-based NGS assays which simultaneously sequence a targeted set of genes with recurrent alterations, associated with known clinical or biological implications are cheaper, less challenging in terms of interpretation and more suited to clinical diagnostics than current approaches [25]. Despite this, development and validation of high throughput gene panel sequencing is challenging. Typically, DNA is only available from formalin-fixed, paraffin-embedded (FFPE) samples, which yields relatively poor quality DNA. DNA extraction and library construction to clinical laboratory standards requires optimisation, and it is necessary to construct a standardised informatics pipeline that identifies and interprets actionable mutations. Appropriate and rapid clinical reporting of identified variants and incorporation of the results into the electronic patient records also need to be considered if molecular stratification of childhood cancer is to be successfully translated to the clinic [26]. There are several examples of validation and implementation of targeted sequencing in adult cancer [27-30]. In the past two years, several approaches using highthroughput sequencing have been applied for clinical decision-making in children with solid tumours [3134], however a clinically validated panel specifically targeting recurrent alterations in childhood cancers using archival FFPE specimens would significantly assist the development of molecular stratification strategies in paediatric oncology.

Here we describe the development and validation, within an accredited clinical pathology laboratory (CPA UK), of a paediatric solid tumour sequencing panel for use with either routine FFPE or fresh frozen (FF) samples. As part of the validation, we established overall performance, sensitivity, specificity, repeatability, reproducibility, accuracy and limit of detection, following guidelines previously described for validation of genetic tests [35]. 


\section{RESULTS}

\section{Selection of panel content}

The panel design covers a total of 78 genes (Table 1), either recurrently altered in paediatric cancers or clinically actionable in adult cancers and with potential application in childhood solid tumours. The genes were selected in widecollaboration with national experts in paediatric oncology patient care covering all areas of paediatric solid tumours (glioma, medulloblastoma, bone sarcomas, soft tissue sarcomas, renal tumours and neuroblastoma among others). Targets were chosen by consensus based on most clinically relevant aberrations including: i) predictive biomarker (level 1), prognostic biomarker (level 2), diagnostic biomarker (3) potentially targetable biomarkers with inhibitors available or under development (level 4), known germline or high risk single nucleotide polymorphism (level 5) or unclear significance, research only (level 6). Factors influencing the choice of targets included: childhood tumour type where alterations have been reported, molecules targeting these genes and clinical trials available for children with solid tumours (Supplementary Table 1A). A library of customized biotinylated DNA probes was designed to capture a total of $\sim 311$ (kilobase) $\mathrm{kb}$ for the detection of single nucleotide variants (SNVs), short insertion-deletions (indels), copy number variations and structural rearrangements (Supplementary Table 1B). Exons were padded with 5 base pairs (bp) of intronic sequence to increase exon depth and for detection of splice-site variants.

\section{Panel validation}

Research use of sequence capture assays has become common, but basing clinical care on gene panel sequencing results requires confident calling of both variant and non-variant sequence, and a full understanding of the performance of the assay. Implementation in the clinic therefore requires robust validation in an accredited laboratory.

To validate the paediatric gene panel, we followed the standardised framework for clinical assay validation set out by Mattocks et al. [35]. We determined overall performance of the panel across the target regions, measuring precision, sensitivity and specificity. As a standard, we used a set of four Horizon cell blends previously characterized by NGS and droplet digital PCR (ddPCR) (Supplementary Table 2A and 2B) and 15 paediatric cell lines with known variants. For further validation, we performed capture and sequencing on 83 FFPE and 30 FF clinical samples (Supplementary Table 3).

\section{Overall performance}

Overall, the panel performed well, with over $96 \%$ of 901 regions of interest achieving specification. Only
$24(2.7 \%)$ regions were classified as underperforming across the four cell blends and five FFPE samples, with read depth lower than $2 \times$ standard deviation (SD) of the mean based on $\log _{2}$ (Supplementary Table 4A and 4B). 22 of 24 underperforming regions were located within highly GC-enriched regions, which are known to be refractory to efficient hybridization and/or amplification (Figure 1 and Supplementary Table 4C).

Quality and coverage metrics were generated across all samples (Supplementary Tables 5 and 6). The average total number of reads was $8.8 \times 10^{6}\left(\mathrm{SD}=3.1 \times 10^{6}\right)$ for FFPE and $7.9 \times 10^{6}\left(\mathrm{SD}=3 \times 10^{6}\right)$ for high molecular weight (HMW) samples (FF and cell lines). The percentage mapped $(96.1 \pm 3.9$ for FFPE vs $97.3 \pm 2.5$ for high molecular weight samples) and percentage of bases from unique reads on target (45.9 \pm 3 for FFPE vs $42.7 \pm 2.4$ for HMW) was very similar for both FFPE and HMW samples. Duplicates were higher in FFPE samples $(60.2 \%$ for FFPE vs $36.1 \%$ for HMW). The overall mean depth was $698 \pm 365$ for FFPE vs $899 \pm 347$ for HMW (Table 2).

\section{Limit of detection}

To determine the limit of detection, SNVs present in the cell blends at known variant allele frequency (VAF) were used. The pipeline detected all 61 SNVs including 33 SNVs with an expected VAF of 4-5\%. 15/17 expected indels were detected. Of the two indels not detected, one was $18 \mathrm{bp}$ in length at an expected VAF of $4.2 \%$, whilst the other was 2 bp at 5\% VAF (Figure 2 and Supplementary Table 7A). We therefore established a minimum threshold of 5\% VAF in the analysis pipeline, which allows for detection of a heterozygous mutation when $>10 \%$ neoplastic cells are present in the tumour sample.

\section{Assessment of precision}

To measure precision, we took advantage of natural variants present as intrinsic "background" SNVs and indels in the captured regions from the four cell blends. Precision was assessed by comparing the alterations expected with those detected to obtain within runprecision (repeatability), and between run-precision data (intermediate precision). Variants $\leq 5 \%$ in all four blends and within poor performing regions were excluded leaving a total of 528 SNVs (132 variants in 4 blends) and 108 indels (27 indels in 4 blends) for analysis. All of the 528 SNVs and 90 out of $108(83 \%)$ indels were detected (Supplementary Table 8).

\section{Repeatability}

Pairwise correlation of VAF between runs was $r^{2} \geq 0.994$ [95\%CI:0.991-0.996] for SNVs and $r^{2} \geq 0.785$ [95\%CI:0.652-0.919] for indels (Supplementary Figures 1 and 2) indicating that the panel accurately reproduces data from independently prepared samples on the same run. 
Table 1: Gene panel list including $\mathbf{7 8}$ genes recurrently altered in paediatric cancers or clinically actionable

\begin{tabular}{|c|c|c|c|}
\hline ACVR1 & CTNNB1 & IL3 & PPM1D \\
\hline$A K T 1$ & $D D R 2$ & IL6 & PTCH1 \\
\hline$A L K$ & $D D X 3 X$ & KIT & PTEN \\
\hline AMER1 & DICERI & $K M T 2 D$ & PTPN11 \\
\hline$A P C$ & $E G F R$ & $K R A S$ & PTPRD \\
\hline ARIDIA & $E R B B 2$ & $L M O 1$ & $R B 1$ \\
\hline$A R I D I B$ & $E R G$ & $M A P 2 K 1$ & RET \\
\hline$A S X L 1$ & ETV6 & $M A P 2 K 2$ & ROS1 \\
\hline$A T M$ & EWSR1 & $M D M 2$ & SETD2 \\
\hline$A T R X$ & $F B X W 7$ & $M Y C N$ & SMARCA4 \\
\hline BARD1 & $F G F R 1$ & MYODI & $S M A R C B 1$ \\
\hline$B C O R$ & $F G F R 2$ & $\mathrm{NCOA} 2$ & SS18 \\
\hline$B R A F$ & $F G F R 3$ & $N F 1$ & SUFU \\
\hline CASC15 & FGFR4 & $N R A S$ & TENM3 \\
\hline$C D K 4$ & FUS & $P A X 3$ & TP53 \\
\hline$C D K 6$ & $H 3 F 3 A$ & $P A X 7$ & TSC1 \\
\hline$C D K N 2 A$ & HIST1H3B & PDGFRA & $W T 1$ \\
\hline$C D K N 2 B$ & HIST1H3C & PHOX $2 B$ & $Z H X 2$ \\
\hline CFL1 & $H R A S$ & $P I K 3 C A$ & \\
\hline CHEK2 & $I D H 1$ & PIK3R1 & \\
\hline
\end{tabular}

\section{Intermediate precision}

Pairwise correlation was $\mathrm{r}^{2} \geq 0.995[95 \% \mathrm{CI}: 0.993-$ 0.997] and overall correlation was $\mathrm{r}^{2}=0.996[95 \% \mathrm{CI}: 0.995$ 0.997] for SNV detection. For indels pairwise correlation was $\mathrm{r}^{2} \geq 0.827 \quad[95 \% \mathrm{CI}: \quad 0.716-0.937]$ and overall correlation was $\mathrm{r}^{2} \geq 0.875$ [95\%CI:0.829-0.921] (Table 3 and Supplementary Figures 3 and 4) indicating that the panel accurately reproduces data from repeat samples on different runs.

\section{Assessment of sensitivity and specificity}

To determine sensitivity we used the same background $528 \mathrm{SNVs}$ and 108 indels, together with the known cancer-specific variants (61 SNVs and 17 indels) from the four cell blends. SNVs and indels were called and their presence was compared to the list of variants expected in the capture regions from the cell blends (Supplementary Tables 7A and 8). All the SNVs were detected, resulting in a sensitivity of $\geq 98 \%$ [95\%CI:0.981]. From the 108 background indels, 18 were not detected, as were 2 of the cancer-specific indels, obtaining a sensitivity of $\geq 83 \%$ [95\%CI:0.761-0.897]. True Positive (TP) of all SNVs $=589$; False-Negative (FN) of all SNVs
$=0$. TPs of all indels $=105 ;$ FNs of all indels $=20$. The undetected indels were manually checked on Integrative Genomics Viewer (IGV). We observed that 12 of 20 were located +4 bp upstream of the exon (our bed file covers \pm 5 bp), four had poor coverage, two fell in highly repetitive regions and one was a long indel (18bp).

To determine specificity, we used the cancer-specific data from the four cell blends harbouring a total of 61 true positive and 87 true negative SNVs (Supplementary Table 7B). There were insufficient true negatives $(n=3)$ to determine specificity for indels. SNVs were called and their presence was compared to the list of variants expected in the capture regions from the cell blends. The specificity of cancer-specific SNVs was $\geq 98 \%$ [95\%CI:0.946-1]. Positive-Predictive Value (PPV) was $\geq 98 \%$ [95\%CI:0.926-1] and the Negative-Predictive Value (NPV) was $\geq 98 \%$ [95\%CI:0.946-1].

The range of VAF for the SNVs detected by our pipeline, including the background and the cancer specific variants $(528+61=589)$, was $23 \%$ at $\geq 50 \%$ VAF (134/589), $35 \%$ at $50-20 \%$ of VAF (207/589) and $42 \%$ at $<20 \%(248 / 589)$. The range of VAF for the indels detected by our pipeline including the background and cancer specific indels $(90+15=105)$ was $0 \%$ at $>50 \%$ of $\operatorname{VAF}(0 / 105), 31 \%$ at $50-20 \%$ of VAF (33/105) and $69 \%$ 
$<20 \%(72 / 105)$. The range of VAF for the cancer specific variants detected by the NGS panel was in line with the manufacturer's specifications.

\section{Performance and variant detection comparison in paired FF-FFPE clinical samples}

To assess the performance of the panel on real clinical material we compared 15 paired clinical DNA samples isolated from both FF and FFPE samples. For the FFPE samples, we obtained an average of $93.4 \% \pm$ $5.42 \%$ and $80.3 \% \pm 20.3 \%$ of targeted positions covered at depths of $\geq 100 \mathrm{x}$ and $\geq 250 \mathrm{x}$ respectively. The overall mean depth for FFPE was $785 \pm 333$. Overall percentage of bases from unique reads on target for FFPE was $47.6 \%$ $\pm 2.3 \%$. For FF samples, we obtained an average of $96.6 \%$ $\pm 0.6 \%$ and $90.9 \% \pm 1.9 \%$ of targeted positions covered at depths of $\geq 100 \mathrm{x}$ and $\geq 250 \mathrm{x}$ respectively. The overall mean depth for FF was $977 \pm 142$. Overall percentage of bases from unique reads on target for FF was $44 \% \pm$ $2.2 \%$. As expected, duplicates were substantially lower in FF samples ( $54.5 \%$ for FFPE vs $29.9 \%$ for FF). Insert size for the library pre-capture DNA was $285 \mathrm{bp} \pm 24$ for FFPE and $326 \mathrm{bp} \pm 24$ for FF (Table 4).

VAFs found in the paired FF-FFPE samples were compared, obtaining an overall correlation of $\mathrm{r}^{2}=$ 0.983 (95\%CI: 0.984-0.985; p<0.0001) (Figure 3 and Supplementary Figure 5). A total of $42.3 \%$ (5562/13146) variants were detected in FF but not in FFPE, of which $78.1 \%(4346 / 5562)$ had VAF below $5 \%$, with $17.6 \%$ (982/5562) having VAF between $5-10 \%$. Less than $5 \%$ variants missed in FFPE samples were present in FF at VAF above $10 \%$.

Conversely, a total of $8.2 \%(1084 / 13146)$ variants were detected in FFPE but not in FF, of which 50.8\%
(551/1084) had VAF below 5\%, with 33.2\% (360/1084) having VAF between $5-10 \%$, and the remaining $16.0 \%$ $(173 / 1084)$ were present in FFPE only at VAF above $10 \%$.

\section{Detection of known variants in paediatric samples}

To assess the ability of the panel to detect known variants in clinical samples, we performed a variant analysis of 41 paediatric samples with 90 known genetic abnormalities (30 alterations in 13 cell lines and 60 alterations in 14 FFPE and 14 FF samples with known genetic alterations identified by routine testing): $50 \mathrm{SNVs}$, including mutations in TP53, ALK, CTNNB1, DDX3X, SMARCA4, one duplication (BRAF p.Thr599dup), 7 indels including $D D X 3 X$ and TP53, 13 amplifications including $M Y C N$ and $C D K 4$, and 19 chromosome/gene losses, for example chr 9q loss including loss of PTCH1 and TSC1. $100 \%$ of the variants interrogated by the panel were successfully detected (Tables 5 and 6 and Supplementary Table 9).

\section{Detection of rearrangements}

Five sarcoma FFPE samples were included in the analysis where translocations had previously been detected by RT-qPCR involving EWSR1. Rearrangements in EWSR1 were detected in four out of the five FFPE samples (80\%) leading to fusion genes of EWSR1 with partners ATF1 (detected in two samples), FLI1 and CREB1 (Supplementary Figure 6). The fusion not detected was EWSR1-NR4A3. This is too small a sample to confirm validation of the panel for detection of translocations at this stage and further work is in progress.

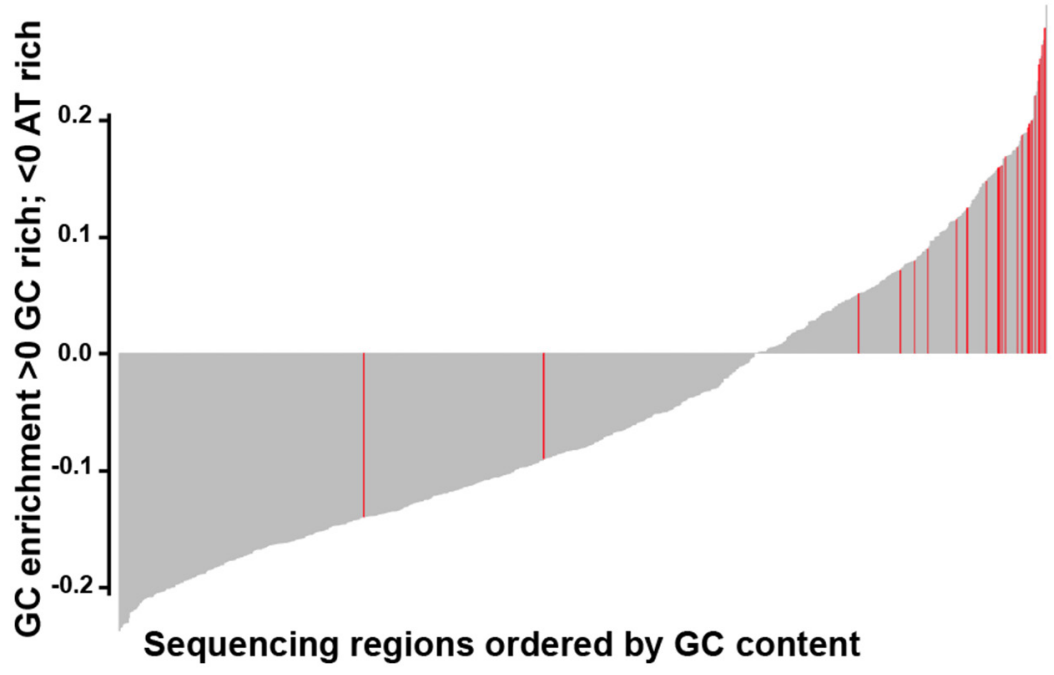

Figure 1: Bar plot showing GC content in the 901 regions captured by the panel. The plot is ordered from low to high GCcontent of each region captured. Red bars highlight the underperforming regions (24/901), mainly located within GC-enriched regions. 
Table 2: Average quality metrics across all samples. Data expressed as means \pm standard deviation

\begin{tabular}{|c|c|c|c|c|c|}
\hline & Total reads & $\begin{array}{l}\text { Percentage of } \\
\text { reads mapped }\end{array}$ & $\begin{array}{c}\text { Percentage of } \\
\text { duplicates }\end{array}$ & $\begin{array}{c}\text { Percentage of } \\
\text { unique on target }\end{array}$ & Mean depth \\
\hline $\operatorname{FFPE}(\mathrm{n}=83)$ & $8.8 \times 10^{6} \pm 3.1 \times 10^{6}$ & $96.1 \pm 3.9$ & $60.2 \pm 13.7$ & $45.9 \pm 3$ & $698 \pm 365$ \\
\hline $\begin{array}{l}\text { FF and cell lines } \\
(n=49)\end{array}$ & $7.9 \times 10^{6} \pm 3 \times 10^{6}$ & $97.3 \pm 2.5$ & $36.1 \pm 9.7$ & $42.7 \pm 2.4$ & $899 \pm 347$ \\
\hline
\end{tabular}

\section{DISCUSSION}

Targeted therapies are already the standard of care for several molecular subgroups of adult cancers. $E G F R$ mutations or $A L K$ rearrangements in lung cancer, $B R A F$ V600E mutations in metastatic melanoma and breast cancer patients harbouring HER2 amplifications are examples of therapeutic biomarkers routinely used in the adult population [36-38]. The implementation of personalised medicine in paediatric oncology has remained challenging partly due to the low incidence of childhood cancer, accessibility of drugs and regulatory hurdles [39]. Nevertheless, the understanding of genetics in childhood cancer over the last decade has improved thanks to large sequencing initiatives across the world [31-33]. The updated World Health Organization Classification (WHO) classification of brain tumours based on molecular features is a clear example of the huge impact of applying molecular profiling to guide diagnosis and treatment with the potential to improve outcomes in childhood cancers [40].

We have developed an NGS targeted sequencing based diagnostic test to accurately detect clinically relevant genomic alterations across 78 cancer genes in routine FFPE as well as FF paediatric samples. The overall performance of our assay was excellent; from the 901 regions captured only $24(<3 \%)$ failed the quality control metrics mainly as a result of being located in GCrich regions, and should be noted for future panel design. VAF for known SNVs and indels were very similar in within-run and between-run replicates, demonstrating that the assay is repeatable and reproducible. SNVs were detected at a wide range of VAFs simulating the heterogeneity expected in cancer samples including 33 SNVs with an expected VAF of $4-5 \%$. The detection of variants at low VAF is crucial, especially in samples with a low neoplastic cell content. Sensitivity was $\geq 98 \%$ for SNVs and $\geq 83 \%$ for indels and specificity $\geq 98 \%$ for SNVs. False-negative calls were mostly indels at low VAF $(\leq 5 \%)$ and predominantly occurred at splice sites. Variants were analysed in exons and the surrounding 5 bp, but were not reported by our pipeline if they occurred outside \pm 2 bp of the coding region. This could be solved expanding the sequence covered by bed file at intron:exon boundaries, but the relevance of these variants remains unclear. There is currently no consensus as to the most

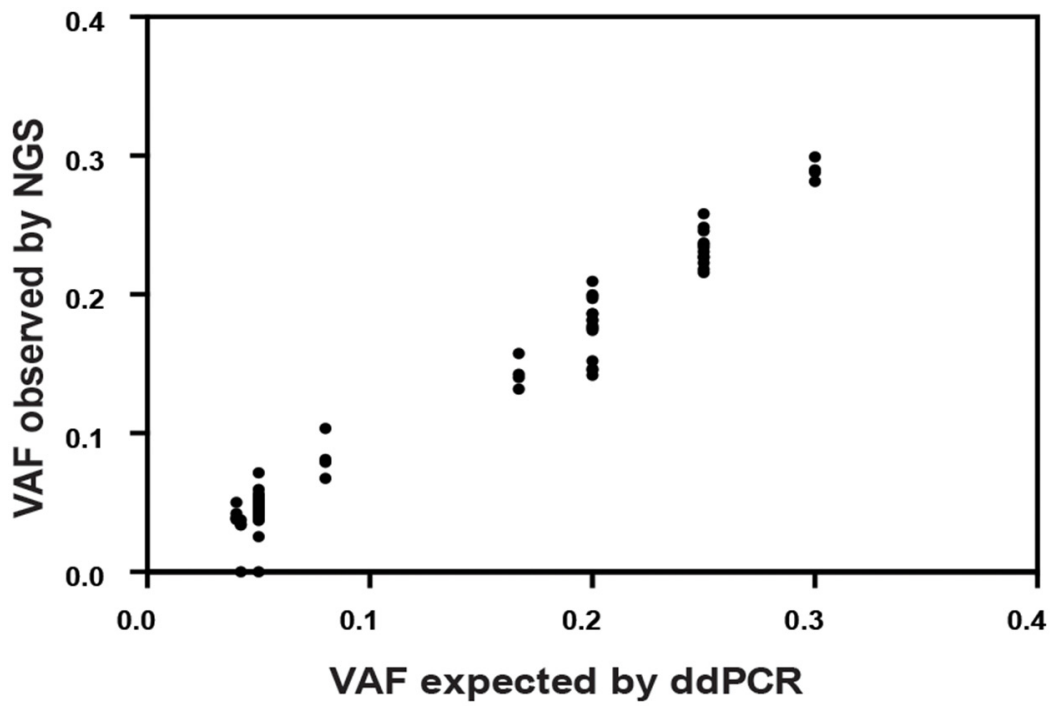

Figure 2: Comparison of known variant allele frequencies by droplet digital PCR (x axis) against variant allele frequency obtained by NGS (y axis) for all cancer-specific variants (61 single nucleotide variants, SNVs and 17 insertion-deletions, indels). Overall correlation was $\mathrm{r}^{2}=0.969$ [95\% CI: 0.975-0.990; $\left.\mathrm{p}<0.0001\right]$. 
Table 3: Pairwise correlation of (A) single nucleotide variants (SNVs) and (B) insertion-deletions (indels) for each of the 4 cell blends with identical background variants between the two runs

\begin{tabular}{lcccc}
\hline A Samples & Correlation & Standard Error & Lower 95\%CI & Upper 95\%CI \\
\hline Tru-Q1-HD728-T & 0.995 & 0.001 & 0.993 & 0.997 \\
Tru-Q2-HD729-T & 0.996 & 0.001 & 0.995 & 0.997 \\
Tru-Q3-HD730-T & 0.996 & 0.001 & 0.995 & 0.998 \\
Tru-Q4-HD731-T & 0.997 & 0.001 & 0.995 & 0.998 \\
\hline B Samples & Correlation & Standard Error & Lower 95\%CI & Upper 95\%CI \\
\hline Tru-Q1-HD728-T & 0.912 & 0.03 & 0.853 & 0.971 \\
Tru-Q2-HD729-T & 0.905 & 0.032 & 0.842 & 0.969 \\
Tru-Q3-HD730-T & 0.858 & 0.047 & 0.766 & 0.951 \\
Tru-Q4-HD731-T & 0.827 & 0.057 & 0.716 & 0.937 \\
\hline
\end{tabular}

appropriate minimum region of interest to cover at splice sites for clinical reporting and in many cases the biological meaning of these mutations are unknown. The latest guidelines recommend calling likely disrupted gene function in nonsense, frameshift, canonical \pm 1 or \pm 2 splice sites, initiation codon, and single exon or multi-exon deletion, all of which would be covered with our current pipeline [41].

We also compared the performance of paired FFPEFF specimens obtaining comparable quality metrics between both tissue types, as well as a high overall correlation of VAF. This is particularly important as most clinical samples routinely available are derived from FFPE tissue where nucleic acid quality is generally compromised and chemically challenged, leading to DNA degradation and potential deamination or oxidation artefacts. The discrepancies of the variants observed between FFPE and FF were mainly at low VAF, below or at the lower limit of detection of our approach. The discrepancies of the variants above $10 \%$ could be explained by variation in neoplastic cell content between FF and FFPE and intratumour heterogeneity leading to sub-clonal alterations. Three of the samples with more striking differences were brain tumours which are well known as highly heterogeneous tumours [42, 43].

We verified the accuracy of our NGS approach in cell lines and clinical specimens (FFPE and FF) containing known genetic abnormalities previously characterized by other methodologies and obtained a high concordance $\left(\mathrm{r}^{2}=\right.$ 0.983). The FFPE and FF samples used for the validation were a cohort of specimens from several hospitals across the world. We obtained reproducible and accurate results

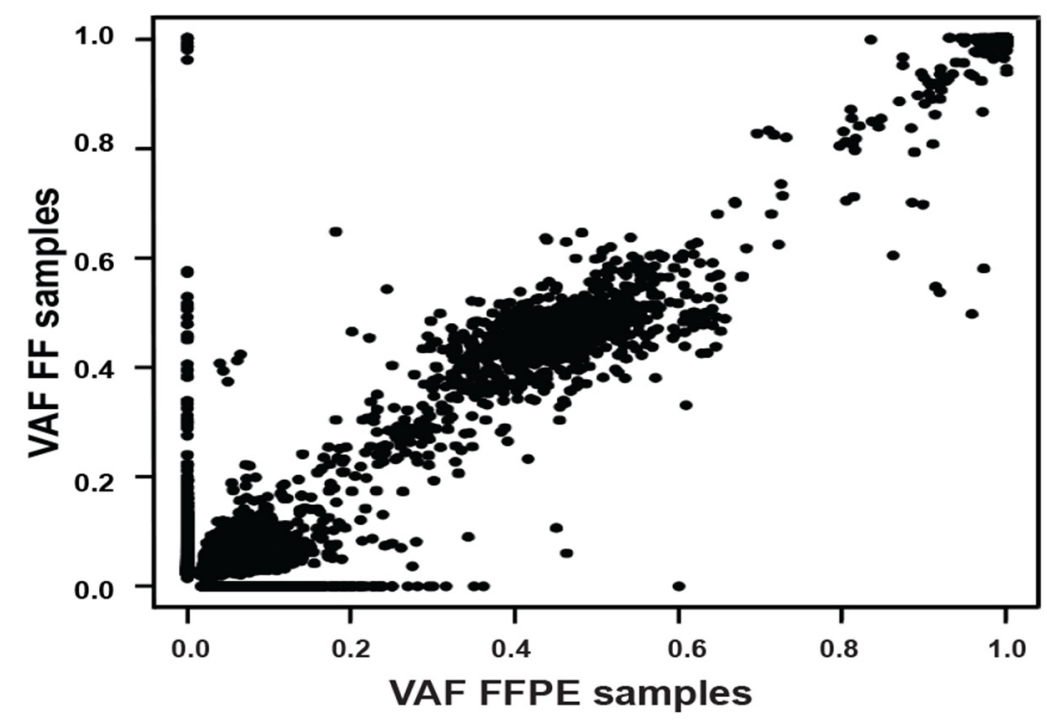

Figure 3: Overall correlation of variant allele frequency (VAFs) found between the 15 formalin-fixed paraffin embedded ( $x$ axis) and fresh frozen ( $y$ axis) paired samples. 
Table 4: Comparison of quality metrics between formalin-fixed paraffin embedded (FFPE) and fresh frozen (FF) matched samples $(n=15)$

\begin{tabular}{lccccc}
\hline & Total reads & $\begin{array}{c}\text { Percentage of } \\
\text { reads mapped }\end{array}$ & $\begin{array}{c}\text { Percentage of } \\
\text { duplicates }\end{array}$ & $\begin{array}{c}\text { Percentage of } \\
\text { unique on target }\end{array}$ & Mean depth \\
\hline FFPE $(\mathrm{n}=15)$ & $8.7 \times 10^{7} \pm 3.4 \times 10^{6}$ & $95.5 \pm 2.2$ & $54.5 .2 \pm 9.2$ & $47.6 \pm 2.3$ & $785 \pm 333$ \\
$\mathrm{FF}(\mathrm{n}=15)$ & $7.7 \times 10^{6} \pm 1.2 \times 10^{6}$ & $98.6 \pm 0.7$ & $29.9 \pm 6.9$ & $44.2 \pm 2.2$ & $977 \pm 142$ \\
\hline
\end{tabular}

Data expressed as means \pm standard deviation.

Table 5: Known variants in paediatric cancer cell lines were compared against capture sequencing from the Cancer Cell Line Encyclopaedia and other published data

\begin{tabular}{|c|c|c|c|c|c|}
\hline Cell line ID & Gene & Alteration & Detected & $\begin{array}{c}\text { Allele frequency } \\
\text { expected }\end{array}$ & Allele frequency observed \\
\hline $\operatorname{Be}(2) \mathrm{C}$ & TP53 & p.C135F & YES & no data available & $100 \%$ \\
\hline $\operatorname{Be}(2) \mathrm{C}$ & MYCN & AMPLIFICATION & YES & not applicable & not applicable \\
\hline $\mathrm{CCA}$ & KRAS & p.Q61L & YES & no data available & $29 \%$ \\
\hline IMR32 & ATM & p.V2716A & YES & $59 \%$ & $59 \%$ \\
\hline IMR32 & MYCN & AMPLIFICATION & YES & not applicable & not applicable \\
\hline KELLY & ALK & p.F1174L & YES & $39 \%$ & $32 \%$ \\
\hline KELLY & MAP2K1 & p.A390T & YES & $48 \%$ & $47 \%$ \\
\hline KELLY & TP53 & p.P177T & YES & $93 \%$ & $99 \%$ \\
\hline KELLY & MYCN & AMPLIFICATION & YES & not applicable & not applicable \\
\hline LAN1 & ALK & p.F1174L & YES & no data available & $47 \%$ \\
\hline LAN1 & TP53 & p.C182* & YES & no data available & $99 \%$ \\
\hline LAN1 & MYCN & AMPLIFICATION & YES & not applicable & not applicable \\
\hline LAN5 & ALK & p.R1275Q & YES & no data available & $50 \%$ \\
\hline LAN5 & MYCN & AMPLIFICATION & YES & not applicable & not applicable \\
\hline NBLS & NF1 & $\begin{array}{l}\text { splice_acceptor_ } \\
\text { variant c.6705-1G>T }\end{array}$ & YES & no data available & $42 \%$ \\
\hline $\mathrm{RD}$ & $\mathrm{ATM}^{\dagger}$ & p.D273N & YES & $17 \%$ & $2 \%$ \\
\hline $\mathrm{RD}$ & NF1 & p.E977* & YES & $56 \%$ & $59 \%$ \\
\hline $\mathrm{RD}$ & NRAS & p.Q61H & YES & $68 \%$ & $61 \%$ \\
\hline $\mathrm{RD}$ & TP53 & p.R248W & YES & $100 \%$ & $100 \%$ \\
\hline RH30 & CDK4 & AMPLIFICATION & YES & not applicable & not applicable \\
\hline RH41 & APC & p.M526L & YES & $60 \%$ & $59 \%$ \\
\hline RH41 & TP53 & p.P152fs & YES & $100 \%$ & $100 \%$ \\
\hline RMS559 & FGFR4 & p.V582L & YES & no data available & $76 \%$ \\
\hline SKNAS & NRAS & p.Q61L & YES & $45 \%$ & $46 \%$ \\
\hline SKNAS & RB1 & p.L477P & YES & $47 \%$ & $31 \%$ \\
\hline SKNAS & TP53 & DEL exons 10,11 & YES & not applicable & not applicable \\
\hline SKNSH & NRAS & p.Q61L & YES & $15 \%$ & $23 \%$ \\
\hline SKNSH & SMARCA4 & p.R973T & YES & $32 \%$ & $45 \%$ \\
\hline SKNSH & CHEK2 & p.T410fs & YES & $59 \%$ & $44 \%$ \\
\hline SKNSH & ALK & p.F1174L & YES & no data available & $36 \%$ \\
\hline
\end{tabular}

${ }^{\dagger} A T M$ mutation in this cell line is subclonal and variation in AF is expected with on-going passages. 
Table 6: Known variants in paediatric FFPE $(n=14)$ and FF $(n=14)$ samples were compared against other platforms such as RNA seq, 450k array, Sanger Sequencing and FISH

\begin{tabular}{|c|c|c|c|c|}
\hline $\begin{array}{l}\text { Genes with alterations detected by other } \\
\text { methodologies }\end{array}$ & Alteration & Tumour type & $\begin{array}{l}\text { Total cases } \\
\text { expected }\end{array}$ & $\begin{array}{l}\% \text { of cases } \\
\text { detected }\end{array}$ \\
\hline DDX3X & SNV and indel & Medulloblastoma & 6 & 100 \\
\hline PTCH1 & SNV and indel & Medulloblastoma & 5 & 100 \\
\hline TP53 & SNV and indel & Medulloblastoma & 3 & 100 \\
\hline MYCN & SNV & Medulloblastoma & 2 & 100 \\
\hline MYCN & Amplification & $\begin{array}{l}\text { Neuroblastoma }(\mathrm{n}=3) \\
\text { Medulloblastoma } \\
(\mathrm{n}=4)\end{array}$ & 7 & 100 \\
\hline CTNNB1 & SNV & Medulloblastoma & 5 & 100 \\
\hline H3F3A & SNV & Glioma & 3 & 100 \\
\hline SMARCA4 & SNV & Medulloblastoma & 3 & 100 \\
\hline BRAF & SNV & Glioma & 2 & 100 \\
\hline ALK & SNV & Neuroblastoma & 1 & 100 \\
\hline HIST1H3B & SNV & Glioma & 1 & 100 \\
\hline AKT1 & SNV & Medulloblastoma & 1 & 100 \\
\hline ACVR1 & SNV & Medulloblastoma & 1 & 100 \\
\hline PIK3CA & SNV & Medulloblastoma & 1 & 100 \\
\hline MLL2 & SNV & Medulloblastoma & 1 & 100 \\
\hline chr 9q - (PTCH1, TSC1) & loss & Medulloblastoma & 5 & 100 \\
\hline chr 10- (PTEN, SUFU, FGFR2) & loss & Medulloblastoma & 4 & 100 \\
\hline chr 6- (HIST1H3B, HIST1H3C, ROS1, ARID1B) & loss & Medulloblastoma & 2 & 100 \\
\hline chr 9- (CDKN2A/B, PTHC1, TSC1) & loss & Medulloblastoma & 2 & 100 \\
\hline chr12- (MLL2, CDK4) & loss & Medulloblastoma & 1 & 100 \\
\hline ATM LOH & loss & Medulloblastoma & 1 & 100 \\
\hline chr 3p- (CTNNB1, STED2) & loss & Medulloblastoma & 1 & 100 \\
\hline chr17- (TP53, NF1, HER2, PPM1D) & loss & Medulloblastoma & 1 & 100 \\
\hline chr17p- (NF1, TP53) & loss & Medulloblastoma & 1 & 100 \\
\hline Total & & & 60 & \\
\hline
\end{tabular}

from different quality samples processed in different pathology laboratories, demonstrating the value of this approach for the development of national and international clinical trials in paediatric oncology.

Our data shows that this NGS approach can detect structural variants, including amplifications, deletions and chromosomal rearrangements. These types of variants are not generally detected with commercial amplicon-based NGS panels, despite being of critical importance for the clinical management and diagnosis of paediatric patients (e.g. MYCN amplification in neuroblastoma, EWSR1 in Ewing's sarcoma). Only one out of five chromosomal rearrangements involving EWSR 1 was not identified by the assay which could be due to the lack of coverage at the intronic genomic location of the breakpoint. As expected, this is one of the limitations of the methodology, as capturing intronic regions commonly involved in translocations poses challenges associated to the presence of repetitive sequence elements. This can be partially overcome by including capture baits for the breakpoint regions of the most common partner genes involved in the translocations.

In summary, we have developed a robust clinical test that can detect SNVs, small indels, copy number variation and with high reproducibility and repeatability in routine clinical FFPE samples from a variety of centres. 
Our approach has been incorporated into a pilot molecular profiling study for paediatric patients at the Royal Marsden Hospital (London, UK) and this has now been extended across the UK as the METEOR programme, an interim step towards the UK's more advanced paediatric molecular profiling programme, Stratified MedicinePaediatrics (SM-Paeds) which is about to be rolled out throughout the UK. The NGS panel will form a key part of the SM-Paeds programme, which is underpinning UK patient eligibility screening for several clinical trials including the highly innovative international ITCC basket trial, called ESMART (NCT02813135), where patients are enrolled according to molecular alterations found in their tumours on biopsy at relapse. This is the first time that genomic results are incorporated into the patient's record in paediatric cancer in the UK within a clinically relevant timeframe of 3-5 weeks from DNA extraction to report generation. Our data shows that this NGS assay can be an accurate and a practical platform for molecular stratification and identification of actionable targets required to accelerate personalised medicine clinical trials in childhood solid tumours.

\section{MATERIALS AND METHODS}

\section{Validation samples}

A representative selection of common, poor risk paediatric tumours was used for the validation comprising 132 samples: i) Four cell blends with validated variants (Tru-Q1-4 HorizonDiscovery, Cambridge, UK), ii) 15 paediatric cell lines iii) 83 FFPE clinical samples and iv) 30 FF clinical samples (Supplementary Table 3).

Local institutional review board approval was obtained for the project in addition to separate approvals from the contributing tumour banks (The Children's Cancer and Leukaemia Group Tumour Bank and the Queensland Children's Tumour Bank).

\section{Sample preparation}

Assessment from haematoxylin and eosin (H\&E) stained slides was performed by experienced pathologists to mark the region of the section containing tumour and to estimate neoplastic cell content, defined as the percentage of neoplastic cells out of total nucleated cells in the marked area. Tumour cellularity, reflecting the density of tumour nuclei, was also estimated. Macro-dissection of the marked area was performed when a distinct area of neoplastic cells from normal cells was observed in a large area and the overall tumour content without macrodissection would have been $<60 \%$. 24 out of the 83 FFPE samples underwent macro-dissection to enrich the tumour content. DNA from blood and cell lines, FF and FFPE samples was extracted using the QIAamp DNA blood mini kit, the QIAamp DNA mini kit and the QIAamp DNA
FFPE tissue kit (Qiagen, Hilden, Germany), respectively. For specimens where DNA was extracted at local centres, methods are provided in Supplementary Materials. DNA was quantified using Qubit dsDNA High Sensitivity Assay Kit with the Qubit 2.0 fluorometer, (Invitrogen, Carlsbad, CA). Analysis by TapeStation 2200 using the genomic DNA ScreenTape assay (Agilent Technologies, Santa Clara, CA) was performed to determine the degree of fragmentation of genomic DNA prior to library preparation. Based on optimization studies, samples yielding DNA with median fragment length $>1000 \mathrm{bp}$ were processed using $200 \mathrm{ng}$ DNA. Samples with DNA $<1000$ bp were processed using $400 \mathrm{ng}$ if there was sufficient DNA.

\section{Gene panel capture and sequencing}

Library preparation was performed using the KAPA Hyper and HyperPlus Kit (Kapa Biosystems, Wilmington, MA, USA) and SeqCap EZ adapters (Roche, NimbleGen, Madison WI, USA), following the manufacturer's protocol, including dual-SPRI size selection of the libraries (250-450 bp). In samples prepared using the KAPA Hyper Kit $(n=39)$, DNA was sheared with the Covaris M220 (Covaris, Woburn, MA) using supplier protocols. KAPA HyperPlus employs enzymatic fragmentation and was used in 93 samples. Optimization of the process indicated that the change from enzymatic fragmentation resulted in a substantial improvement in library complexity and unique coverage depth compared to sonication [44]. Following fragmentation DNA was end-repaired, A-tailed and indexed adapters ligated. To optimise enrichment and reduce off-target capture, pooled, multiplexed, amplified pre-capture libraries (6 to 10 cycles according to the DNA input) were hybridized twice overnight (up to 13 samples per hybridization, consecutive days) using $1 \mu \mathrm{g}$ of the pooled library DNA to a custom design of DNA baits complementary to the genomic regions of interest (NimbleGen SeqCap EZ library, Roche, Madison, WI, USA). A 5 cycle PCR was performed between hybridizations to enrich the captured product. After hybridisation, unbound capture baits were washed away and the remaining hybridised DNA was PCR amplified (12 cycles). PCR products were purified using AMPure XP beads (Beckman Coulter, Danvers, MA, USA) and quantified using the KAPA Quantification q-PCR Kit (KAPA Biosystems, Wilmington, MA, USA). Sequencing was performed on a MiSeq (Illumina, San Diego, CA, USA) with 75 bp paired-end reads and v3 chemistry according to the manufacturer's instructions. For samples where germline matched control was available $(n=23)$, pools from tumour and control DNA libraries were multiplexed separately for hybridization and combined prior to sequencing at a ratio of $4: 1$, increasing the relative number of reads derived from tumour DNA. 


\section{Data analysis}

Primary analysis was performed using MiSeq Reporter Software (v2.5.1; Illumina), generating nucleotide sequences and base quality scores in Fastq format. Resulting sequences were aligned against the human reference sequence build GRCh37/Hg19 to generate binary alignment (BAM) and variant call files (vcf). Secondary analysis was performed in-house using Molecular Diagnostics Information Management System to generate QC, variant annotation, data visualisation and a clinical report. In the Molecular Diagnostics Information Management System, reads were deduplicated using Picard (http://broadinstitute.github.io/picard/), and metrics generated for each panel region. Oncotator (v1.5.3.0) (https://portals.broadinstitute.org/oncotator) was used to annotate point mutations and indels using a minimum variant allele frequency (VAF) of 5\% and a minimum number of 10 variant reads. Manta (https://github.com/ Illumina/manta) was used for the detection of structural variants. Variants were annotated for gene names, nature of variant (e.g. missense), PolyPhen-2 predictions, and cancer-specific annotations from the variant databases including COSMIC, Tumorscape, and published MutSig results. Copy number variation (CNV) was assessed using the ratio of GC-normalized depth of region of interest (ROI) in tumour against GC-normalized read depth of ROI in either matched germline DNA (when available) or the male cell line G147A (Promega, Madison, WI USA). Any ratio below 0.65 fold was defined as a potential deletion whereas a ratio above 2.4 was flagged as a potential amplification. All potential mutations, structural variants and CNVs were visualised using IGV and two individuals were required to review the mutation report independently. Variant calls from samples with previously known SNVs and indels were checked manually on IGV.

\section{Cell blends}

The four cell blends contained 163 SNVs and 34 indels common to all four blends (background variants) (Supplementary Table 2A). Additionally, there were 61 SNVs and 17 indels, cancer variants, which were unique between blends, introduced at known VAF, and verified by ddPCR (Supplementary Table 2B). The four cell blends were used to assess overall performance, repeatability, intermediate precision, sensitivity and limit of detection. Specificity was determined using 87 true negative SNV sites (wild type) where another blend harboured a mutation at the corresponding position. The cell blends were processed and sequenced in two different runs by two independent users.

\section{Overall performance}

Four cell blends and five FFPE samples were used to measure performance across the capture design.
The log mean depth across the panel was compared to the $\log$ depth of each region captured for each gene. Regions were classified as underperforming if the depth was lower than $2 \times$ SD of the mean based on $\log _{2}$ $\left[\log _{2}(\mathrm{ROI})>\operatorname{mean}\left(\log _{2}(\mathrm{ROI})\right)-2 \mathrm{xSD}\left(\log _{2}(\mathrm{ROI})\right)\right] . \quad \mathrm{GC}$ content and mappability scores were compared against each region captured by the panel. Quality and coverage metrics were calculated across all the samples including i) total reads, ii) percentage of reads mapped to the reference sequence, iii) percentage of duplicates, iv) percentage of bases from unique reads de-duplicated on target, v) mean depth of targeted positions and vi) percentage of targeted positions with $\geq 50 \mathrm{x}, \geq 100 \mathrm{x}$ and $\geq 250 \mathrm{x}$ coverage.

\section{Limit of detection}

To assess the limit of detection and determine a reliable cut off for the analysis we used the unique cancerspecific set of variants from the four cell blends introduced at range of VAFs from $4 \%$ to $30 \%$, defined by ddPCR.

\section{Precision}

Repeatability (or within-run precision) was determined by comparing the cell blend background variant data across the 4 different samples in the same run for variant detection and VAF. Intra-run pairwise correlation was calculated for two runs where the cell blends were prepared and sequenced by different users generating two sets of repeatability data.

Intermediate precision (or between-run precision) was determined by comparing the cell blend background variant data between two runs for variant detection and VAF. Between-run pairwise correlation was calculated from two different runs prepared by different users and sequenced on different MiSeq instruments.

\section{Sensitivity and specificity}

The sensitivity of the panel was determined by separately comparing the cell blend background variants and the cancer-specific variants introduced at known VAF. Specificity was determined using the cell blend cancerspecific set of data with known variants and known true negative sites. Variants were classified according to the different ranges of frequencies of the variants present in the DNA blends. We also determined Positive-Predictive Value and Negative-Predictive Value.

\section{Correlation between NGS targeted panel and other methodologies}

13 paediatric cancer cell lines were tested harbouring a total of 30 known SNVs, deletions and amplifications previously identified by the Cancer Cell Line Encyclopaedia using Target Enrichment Sequencing (Agilent Technologies, Santa Clara, CA) and other 
published data [45-50]. Furthermore 33 samples $(F F=14$, $\mathrm{FFPE}=19)$ had a total of 65 known genetic alterations including i) SNVs detected by Sanger Sequencing ( $H 3 F 3 A$, TP53, CTNNB1, HIST1H3B, ALK, BRAF) [51-53] and RNA-Seq ii) copy number changes by FISH (MYCN) [54] and 450k array and iii) rearrangements by RealTime Quantitative PCR involving ESWR1 as previously described [55, 56] (Refer to Supplementary Materials).

\section{Fresh frozen vs FFPE samples}

15 paired FF and FFPE paediatric samples were compared for quality control metrics, coverage and the distribution of library inserts sizes between FFPE and FF paired samples. In addition, we correlated the VAF of the total variants found in the paired samples.

\section{Abbreviations}

FFPE (formalin-fixed paraffin embedded); FF (fresh frozen); SNVs (single nucleotide variants); Indels (insertion-deletions); DIPG (diffuse intrinsic pontine glioma); NGS (next-generation sequencing); kb (kilobase); bp (base pairs); ddPCR (droplet digital PCR); SD (standard deviation); CI (confidential interval); HMW (high molecular weight); VAF (variant allele frequency); TP (true positive); FN (false negative); PPV (positivepredictive Value); NPV (negative-predictive Value); WHO (world health organization); H\&E (haematoxylin and eosin); BAM (binary alignment); vcf (variant call files); CNV (copy number variation); ROI (region of interest); IGV (integrative genomics viewer).

\section{Author contributions}

E Izquierdo designed and performed experiments, analysed-interpreted data, and wrote the manuscript; L Yuan analysed-interpreted the data and constructed analytical and visualisation tools; S George, L Chesler helped with the design, provided samples and gave advice; P Proszek performed experiments; C Jones, J Shipley, SA Gatz, L Marshall, C Stinson, AS Moore, SC Clifford, D Hicks, J Lindsey, R Hill, TS Jacques and J Chalker provided samples and/or gave advice; A Pearson, L Moreno and D Gonzalez de Castro conceived and designed the study; B Walker and D Gonzalez de Castro, supervised the research, interpreted data and reviewed the manuscript; D Gonzalez de Castro, K Thway and SO Connor carried out histopathological analysis; L Moreno reviewed the manuscript; M Hubank reviewed and edited the manuscript. All authors read and approved the final manuscript.

\section{ACKNOWLEDGMENTS}

We are enormously grateful to the Christopher's Smile charity (grant numbers CSM 002 and CSM 003) for their support and enthusiasm to provide children with more effective and less toxic targeted drugs through molecular profiling. We also thank the contribution of Children's Cancer and Leukaemia Group (CCLG).

\section{CONFLICTS OF INTEREST}

The authors declared no conflicts of interest with the submitted paper.

\section{FINANCIAL SUPPORT}

This work was supported by Christopher's Smile charity (CSM 002 and CSM 003) and the the NIHR Biomedical Research Centre at the Royal Marsden and the Institute of Cancer Research in London (Grant ref. A113).

\section{REFERENCES}

1. Siegel R, Naishadham D, Jemal A. Cancer statistics, 2012. CA Cancer J Clin. 2012; 62:10-29.

2. London WB, Castel V, Monclair T, Ambros PF, Pearson AD, Cohn SL, Berthold F, Nakagawara A, Ladenstein RL, Iehara T, Matthay KK. Clinical and biologic features predictive of survival after relapse of neuroblastoma: a report from the International Neuroblastoma Risk Group project. J Clin Oncol. 2011; 29:3286-92.

3. Oberlin O, Rey A, Lyden E, Bisogno G, Stevens MC, Meyer WH, Carli M, Anderson JR. Prognostic factors in metastatic rhabdomyosarcomas: results of a pooled analysis from United States and European cooperative groups. J Clin Oncol. 2008; 26:2384-9.

4. Smith MA, Altekruse SF, Adamson PC, Reaman GH, Seibel NL. Declining childhood and adolescent cancer mortality. Cancer. 2014; 120:2497-506.

5. Middleton G, Crack LR, Popat S, Swanton C, Hollingsworth SJ, Buller R, Walker I, Carr TH, Wherton D, Billingham LJ. The National Lung Matrix Trial: translating the biology of stratification in advanced non-small-cell lung cancer. Ann Oncol. 2015; 26:2464-9.

6. Conley BA, Doroshow JH. Molecular analysis for therapy choice: NCI MATCH. Semin Oncol. 2014; 41:297-9.

7. Pugh TJ, Weeraratne SD, Archer TC, Pomeranz Krummel DA, Auclair D, Bochicchio J, Carneiro MO, Carter SL, Cibulskis K, Erlich RL, Greulich H, Lawrence MS, Lennon NJ, et al. Medulloblastoma exome sequencing uncovers subtype-specific somatic mutations. Nature. 2012; 488:106-10.

8. Shukla N, Ameur N, Yilmaz I, Nafa K, Lau CY, Marchetti A, Borsu L, Barr FG, Ladanyi M. Oncogene mutation profiling of pediatric solid tumors reveals significant subsets of embryonal rhabdomyosarcoma and neuroblastoma with mutated genes in growth signaling pathways. Clin Cancer Res. 2012; 18:748-57. 
9. Jones DT, Hutter B, Jager N, Korshunov A, Kool M, Warnatz HJ, Zichner T, Lambert SR, Ryzhova M, Quang DA, Fontebasso AM, Stutz AM, Hutter S, et al. Recurrent somatic alterations of FGFR1 and NTRK2 in pilocytic astrocytoma. Nat Genet. 2013; 45:927-32.

10. Pugh TJ, Morozova O, Attiyeh EF, Asgharzadeh S, Wei JS, Auclair D, Carter SL, Cibulskis K, Hanna M, Kiezun A, Kim J, Lawrence MS, Lichenstein L, et al. The genetic landscape of high-risk neuroblastoma. Nat Genet. 2013; 45:279-84.

11. Robinson G, Parker M, Kranenburg TA, Lu C, Chen X, Ding L, Phoenix TN, Hedlund E, Wei L, Zhu X, Chalhoub $\mathrm{N}$, Baker SJ, Huether R, et al. Novel mutations target distinct subgroups of medulloblastoma. Nature. 2012; 488:43-8.

12. Alexandrov LB, Nik-Zainal S, Wedge DC, Aparicio SA, Behjati S, Biankin AV, Bignell GR, Bolli N, Borg A, Borresen-Dale AL, Boyault S, Burkhardt B, Butler AP, et al. Signatures of mutational processes in human cancer. Nature. 2013; 500:415-21.

13. Wu G, Diaz AK, Paugh BS, Rankin SL, Ju B, Li Y, Zhu X, Qu C, Chen X, Zhang J, Easton J, Edmonson M, Ma X, et al. The genomic landscape of diffuse intrinsic pontine glioma and pediatric non-brainstem high-grade glioma. Nat Genet. 2014; 46:444-50.

14. Hosoi H, Tanaka Y, Okita H, Miyachi M, Souzaki R, Taguchi T, Koh K, Hanada R, Kato K, Nomura Y, Akiyama M, Oka A, Igarashi T, et al. Recurrent somatic structural variations contribute to tumorigenesis in pediatric osteosarcoma. Nat Commun. 2014; 7:104-12.

15. Huether R, Dong L, Chen X, Wu G, Parker M, Wei L, Ma J, Edmonson MN, Hedlund EK, Rusch MC, Shurtleff SA, Mulder HL, Boggs K, et al. The landscape of somatic mutations in epigenetic regulators across 1,000 paediatric cancer genomes. Nat Commun. 2014; 5:3630.

16. Shern JF, Chen L, Chmielecki J, Wei JS, Patidar R, Rosenberg M, Ambrogio L, Auclair D, Wang J, Song YK, Tolman C, Hurd L, Liao H, et al. Comprehensive genomic analysis of rhabdomyosarcoma reveals a landscape of alterations affecting a common genetic axis in fusionpositive and fusion-negative tumors. Cancer Discov. 2014; 4:216-31.

17. Seki M, Nishimura R, Yoshida K, Shimamura T, Shiraishi Y, Sato Y, Kato M, Chiba K, Tanaka H, Hoshino N, Nagae G, Shiozawa Y, Okuno Y. Integrated genetic and epigenetic analysis defines novel molecular subgroups in rhabdomyosarcoma. Nat Commun. 2015; 6:7557.

18. Castel D, Philippe C, Calmon R, Le Dret L, Truffaux N, Boddaert N, Pages M, Taylor KR, Saulnier P, Lacroix L, Mackay A, Jones C, Sainte-Rose C, et al. Histone H3F3A and HIST1H3B K27M mutations define two subgroups of diffuse intrinsic pontine gliomas with different prognosis and phenotypes. Acta Neuropathol. 2015; 130:815-27.

19. Schwartzentruber J, Korshunov A, Liu XY, Jones DT, Pfaff E, Jacob K, Sturm D, Fontebasso AM, Quang DA,
Tonjes M, Hovestadt V, Albrecht S, Kool M, et al. Driver mutations in histone $\mathrm{H} 3.3$ and chromatin remodelling genes in paediatric glioblastoma. Nature. 2012; 482:226-31.

20. Wu G, Broniscer A, McEachron TA, Lu C, Paugh BS, Becksfort J, Qu C, Ding L, Huether R, Parker M, Zhang J, Gajjar A, Dyer MA, et al. Somatic histone H3 alterations in pediatric diffuse intrinsic pontine gliomas and nonbrainstem glioblastomas. Nat Genet. 2012; 44:251-3.

21. Peifer M, Hertwig F, Roels F, Dreidax D, Gartlgruber M, Menon R, Kramer A, Roncaioli JL, Sand F, Heuckmann JM, Ikram F, Schmidt R, Ackermann S, et al. Telomerase activation by genomic rearrangements in high-risk neuroblastoma. Nature. 2015; 526:700-4.

22. Valentijn LJ, Koster J, Zwijnenburg DA, Hasselt NE, van Sluis P, Volckmann R, van Noesel MM, George RE, Tytgat GA, Molenaar JJ, Versteeg R. TERT rearrangements are frequent in neuroblastoma and identify aggressive tumors. Nat Genet. 2015; 47:1411-4.

23. Hertwig F, Peifer M, Fischer M. Telomere maintenance is pivotal for high-risk neuroblastoma. Cell Cycle. 2016; $15: 311-2$.

24. Jones DT, Jager N, Kool M, Zichner T, Hutter B, Sultan M, Cho YJ, Pugh TJ, Hovestadt V, Stutz AM, Rausch T, Warnatz HJ, Ryzhova M, et al. Dissecting the genomic complexity underlying medulloblastoma. Nature. 2012; 488:100-5.

25. Salto-Tellez M, Gonzalez de Castro D. Next-generation sequencing: a change of paradigm in molecular diagnostic validation. J Pathol. 2014; 234:5-10.

26. Hagemann IS, Cottrell CE, Lockwood CM. Design of targeted, capture-based, next generation sequencing tests for precision cancer therapy. Cancer Genet. 2013; 206:420-31.

27. Cheng DT, Mitchell TN, Zehir A, Shah RH, Benayed R, Syed A, Chandramohan R, Liu ZY, Won HH, Scott SN, Brannon AR, O'Reilly C, Sadowska J, et al. Memorial Sloan Kettering-Integrated Mutation Profiling of Actionable Cancer Targets (MSK-IMPACT): a hybridization capturebased next-generation sequencing clinical assay for solid tumor molecular oncology. J Mol Diagn. 2015; 17:251-64.

28. Frampton GM, Fichtenholtz A, Otto GA, Wang K, Downing SR, He J, Schnall-Levin M, White J, Sanford EM, An P, Sun J, Juhn F, Brennan K, et al. Development and validation of a clinical cancer genomic profiling test based on massively parallel DNA sequencing. Nat Biotechnol. 2013; 31:1023-31.

29. Cottrell CE, Al-Kateb H, Bredemeyer AJ, Duncavage EJ, Spencer DH, Abel HJ, Lockwood CM, Hagemann IS, O'Guin SM, Burcea LC, Sawyer CS, Oschwald DM, Stratman JL, et al. Validation of a next-generation sequencing assay for clinical molecular oncology. J Mol Diagn. 2014; 16:89-105.

30. Pritchard CC, Salipante SJ, Koehler K, Smith C, Scroggins S, Wood B, Wu D, Lee MK, Dintzis S, Adey A, Liu Y, Eaton KD, Martins R, et al. Validation and implementation 
of targeted capture and sequencing for the detection of actionable mutation, copy number variation, and gene rearrangement in clinical cancer specimens. The $\mathrm{J}$ Mol Diagn. 2014; 16:56-67.

31. Mody RJ, Wu YM, Lonigro RJ, Cao X, Roychowdhury S, Vats P, Frank KM, Prensner JR, Asangani I, Palanisamy N, Dillman JR, Rabah RM, Kunju LP, et al. Integrative clinical sequencing in the management of refractory or relapsed cancer in youth. JAMA. 2015; 314:913-25.

32. Worst BC, van Tilburg CM, Balasubramanian GP, Fiesel P, Witt R, Freitag A, Boudalil M, Previti C, Wolf S, Schmidt S, Chotewutmontri S, Bewerunge-Hudler M, Schick M, et al. Next-generation personalised medicine for high-risk paediatric cancer patients - the INFORM pilot study. Eur J Cancer. 2016; 65:91-101.

33. Harris $\mathrm{MH}$, DuBois SG, Glade Bender JL, Kim A, Crompton BD, Parker E, Dumont IP, Hong AL, Guo D, Church A, Stegmaier K, Roberts CW, Shusterman S, et al. Multicenter feasibility study of tumor molecular profiling to inform therapeutic decisions in advanced pediatric solid tumors: the Individualized Cancer Therapy (iCat) study. JAMA Oncol. 2016.

34. Allen CE, Laetsch TW, Mody R, Irwin MS, Lim MS, Adamson PC, Seibel NL, Parsons DW, Cho YJ, Janeway K. Target and agent prioritization for the Children's Oncology Group-National Cancer Institute pediatric MATCH trial. J Natl Cancer Inst. 2017.

35. Mattocks CJ, Morris MA, Matthijs G, Swinnen E, Corveleyn A, Dequeker E, Muller CR, Pratt V, Wallace A. A standardized framework for the validation and verification of clinical molecular genetic tests. Eur J Hum Genet. 2010; 18:1276-88.

36. Lynch TJ, Bell DW, Sordella R, Gurubhagavatula S, Okimoto RA, Brannigan BW, Harris PL, Haserlat SM, Supko JG, Haluska FG, Louis DN, Christiani DC, Settleman J, Haber DA. Activating mutations in the epidermal growth factor receptor underlying responsiveness of non-small-cell lung cancer to gefitinib. N Engl J Med. 2004; 350:2129-39.

37. Chapman PB, Hauschild A, Robert C, Haanen JB, Ascierto P, Larkin J, Dummer R, Garbe C, Testori A, Maio M, Hogg D, Lorigan P, Lebbe C, et al. Improved survival with vemurafenib in melanoma with BRAF V600E mutation. N Engl J Med. 2011; 364:2507-16.

38. Kwak EL, Bang YJ, Camidge DR, Shaw AT, Solomon B, Maki RG, Ou SH, Dezube BJ, Janne PA, Costa DB, VarellaGarcia M, Kim WH, Lynch TJ, et al. Anaplastic lymphoma kinase inhibition in non-small-cell lung cancer. N Engl J Med. 2010; 363:1693-703.

39. Vassal G, Rousseau R, Blanc P, Moreno L, Bode G, Schwoch S, Schrappe M, Skolnik J, Bergman L, BradleyGarelik MB, Saha V, Pearson A, Zwierzina H. Creating a unique, multi-stakeholder Paediatric Oncology Platform to improve drug development for children and adolescents with cancer. Eur J Cancer. 2015; 51:218-24.
40. Louis DN, Perry A, Reifenberger G, von Deimling A, Figarella-Branger D, Cavenee WK, Ohgaki H, Wiestler OD, Kleihues P, Ellison DW. The 2016 World Health Organization Classification of Tumors of the Central Nervous System: a summary. Acta Neuropathol. 2016; 131:803-20.

41. Richards S, Aziz N, Bale S, Bick D, Das S, Gastier-Foster J, Grody WW, Hegde M, Lyon E, Spector E, Voelkerding K, Rehm HL. Standards and guidelines for the interpretation of sequence variants: a joint consensus recommendation of the American College of Medical Genetics and Genomics and the Association for Molecular Pathology. 2015; 17:405-24.

42. Nikbakht H, Panditharatna E, Mikael LG, Li R, Gayden T, Osmond M, Ho CY, Kambhampati M, Hwang EI, Faury D, Siu A, Papillon-Cavanagh S, Bechet D, et al. Spatial and temporal homogeneity of driver mutations in diffuse intrinsic pontine glioma. Nat Commun. 2016; 7:11185.

43. Johnson BE, Mazor T, Hong C, Barnes M, Aihara K, McLean CY, Fouse SD, Yamamoto S, Ueda H, Tatsuno K, Asthana S, Jalbert LE, Nelson SJ, et al. Mutational analysis reveals the origin and therapy-driven evolution of recurrent glioma. Science. 2014; 343:189-93.

44. Appel M, Walker B, Jones T, Izquierdo Delgado E, Raterman D, Rosenbaum H, Burgess D. KAPA HyperPlus/ SeqCap EZ workflow: Improving Data Quality and Turnaround Times for Targeted Next-Generation Sequencing of FFPE DNA. Kapa Biosystems. 2016.

45. Barretina J, Caponigro G, Stransky N, Venkatesan K, Margolin AA, Kim S, Wilson CJ, Lehar J, Kryukov GV, Sonkin D, Reddy A, Liu M, Murray L, et al. The Cancer Cell Line Encyclopedia enables predictive modelling of anticancer drug sensitivity. Nature. 2012; 483:603-7.

46. Hinson AR, Jones R, Crose LE, Belyea BC, Barr FG, Linardic CM. Human rhabdomyosarcoma cell lines for rhabdomyosarcoma research: utility and pitfalls. Front Oncol. 2013; 3:183.

47. Chen Y, Takita J, Choi YL, Kato M, Ohira M, Sanada M, Wang L, Soda M, Kikuchi A, Igarashi T, Nakagawara A, Hayashi Y, Mano H, Ogawa S. Oncogenic mutations of ALK kinase in neuroblastoma. Nature. 2008; 455:971-4.

48. George RE, Sanda T, Hanna M, Frohling S, Luther W 2nd, Zhang J, Ahn Y, Zhou W, London WB, McGrady P, Xue L, Zozulya S, Gregor VE, et al. Activating mutations in ALK provide a therapeutic target in neuroblastoma. Nature. 2008; 455:975-8.

49. McDermott U, Iafrate AJ, Gray NS, Shioda T, Classon M, Maheswaran S, Zhou W, Choi HG, Smith SL, Dowell L, Ulkus LE, Kuhlmann G, Greninger P, et al. Genomic alterations of anaplastic lymphoma kinase may sensitize tumors to anaplastic lymphoma kinase inhibitors. Cancer Res. 2008; 68:3389-95.

50. Eleveld TF, Oldridge DA, Bernard V, Koster J, Daage LC, Diskin SJ, Schild L, Bentahar NB, Bellini A, Chicard M, Lapouble E, Combaret V, Legoix-Ne P, et al. Relapsed 
neuroblastomas show frequent RAS-MAPK pathway mutations. Nat Genet. 2015; 47:864-71.

51. Ellison DW, Onilude OE, Lindsey JC, Lusher ME, Weston CL, Taylor RE, Pearson AD, Clifford SC. betaCatenin status predicts a favorable outcome in childhood medulloblastoma: the United Kingdom Children's Cancer Study Group Brain Tumour Committee. J Clin Oncol. 2005; 23:7951-7.

52. Hill RM, Kuijper S, Lindsey JC, Petrie K, Schwalbe EC, Barker K, Boult JK, Williamson D, Ahmad Z, Hallsworth A, Ryan SL, Poon E, Robinson SP, et al. Combined MYC and P53 defects emerge at medulloblastoma relapse and define rapidly progressive, therapeutically targetable disease. Cancer Cell. 2015; 27:72-84.

53. Bjerke L, Mackay A, Nandhabalan M, Burford A, Jury A, Popov S, Bax DA, Carvalho D, Taylor KR, Vinci M, Bajrami I, McGonnell IM, Lord CJ, et al. Histone H3.3. mutations drive pediatric glioblastoma through upregulation of MYCN. Cancer Discov. 2013; 3:512-9.

54. Lamont JM, McManamy CS, Pearson AD, Clifford SC, Ellison DW. Combined histopathological and molecular cytogenetic stratification of medulloblastoma patients. Clin Cancer Res. 2004; 10:5482-93.

55. Thway K, Wren D, Lee J, Thompson L, Fisher C, Gonzalez D. Evaluation of the optimal provision of formalin-fixed, paraffin-embedded material for reverse transcriptionPCR in soft-tissue tumour diagnosis. J Clin Pathol. 2017; 70:20-4.

56. Thway K, Rockcliffe S, Gonzalez D, Swansbury J, Min T, Thompson L, Fisher C. Utility of sarcoma-specific fusion gene analysis in paraffin-embedded material for routine diagnosis at a specialist centre. J Clin Pathol. 2010; 63:508-12. 